\title{
Abdominal Aorta Thrombosis
}

National Cancer Institute

\section{Source}

National Cancer Institute. Abdominal Aorta Thrombosis. NCI Thesaurus. Code C98701.

Formation of blood clot in the lumen of the abdominal aorta. It may lead to severe abdominal pain and ischemic necrosis of the intestine. 